\title{
A new proof of Euler's pentagonal number theorem
}

\author{
A. David Christopher \\ Department of Mathematics, The American College \\ Tamil Nadu - 625002, India \\ e-mail: davchramedyahoo.co.in
}

Received: 1 March 2017

Accepted: 31 January 2018

Abstract: A new proof of Euler's pentagonal number theorem is obtained.

Keywords: Partitions, Euler's pentagonal number theorem, Jacobi's triple product identity.

2010 Mathematics Subject Classification: Primary 05A17, Secondary 11P81.

\section{History and motivation}

The classical statement of Euler's pentagonal number theorem is

$$
\prod_{n=1}^{\infty}\left(1-q^{n}\right)=\sum_{n=-\infty}^{\infty} q^{\frac{n(3 n-1)}{2}}, \text { where }|q|<1 .
$$

By expanding the left side of the equation (1.1), one can see that

$$
\prod_{n=1}^{\infty}\left(1-q^{n}\right)=1+\sum_{n=1}^{\infty}\left(r_{e}(n)-r_{o}(n)\right) q^{n},
$$

where $r_{e}(n)$ denotes the number of distinct partitions (partitions with distinct parts) of $n$ with even number of parts, and $r_{o}(n)$ denotes the number of distinct partitions of $n$ with odd number of parts.

Equations (1.1) and (1.2) together give the following expression:

$$
r_{e}(n)-r_{o}(n)= \begin{cases}(-1)^{k}, & \text { if } n=\frac{3 k^{2} \pm k}{2} \\ 0, & \text { otherwise }\end{cases}
$$


This expression is known as the partition-theoretic interpretation of Euler's pentagonal number theorem. Euler's pentagonal number theorem follows directly from the Jacobi's triple product identity

$$
\prod_{m=1}^{\infty}\left(1-q^{2 m}\right)\left(1+q^{2 m-1} z^{2}\right)\left(1+q^{2 m-1} z^{-2}\right)=\sum_{n=-\infty}^{\infty} q^{n^{2}} z^{2 n}
$$

for $q=x^{\frac{3}{2}}$ and $z^{2}=-x^{\frac{1}{2}}$.

Applications of Euler's pentagonal number theorem is manifold. Recently, Chuanan Wei and Dianxuan Gong [10] showed that Euler's pentagonal number theorem implies Jacobi's triple product identity. Applying Jacobi's triple product identity, Ewell [6] obtained Fermat's two squares theorem. Hirschhorn [8] obtained Jacobi's two squares theorem as a consequence of Jacobi's Triple Product Identity.

Euler [5] proved the classical version of his theorem using induction. Many mathematicians obtained proofs for Jacobi's triple product identity (for proof see [1, 2, 3, 9, 11]). Addition to these proofs, Franklin [7] gave a bijective proof for Euler's pentagonal number theorem using Ferrer's diagram of the partition, and F. J. Dyson [4] gave a combinatorial proof involving the idea of the rank of a partition.

In this article, we give a new proof for the partition-theoretic version of Euler's pentagonal number theorem.

Definition 1.1. Let $n$ be a positive integer. A partition $\left(a_{1}, a_{2}, \ldots, a_{k}\right)$ of $n$ is said to be a distinct partition of $n$ if $a_{i}>a_{i+1}$ for every $i \in\{1,2, \ldots, k-1\}$.

\section{Proof}

Let $n$ be a positive integer. Let $Q_{n}$ be the set of all distinct partitions of $n$. Define an operator $\phi: Q_{n} \rightarrow Q_{n}$ by

$$
\phi\left(\left(a_{1}, a_{2}, \ldots, a_{k}\right)\right)=\left(a_{1}+1, a_{2}+1, \ldots, a_{a_{k}}+1, a_{a_{k}+1}, \ldots, a_{k-1}\right)
$$

when $a_{k}<k$.

Let $Q_{n, s}$ be the set of all distinct partitions of $n$ with its least part $s$ such that $s<$ number of parts.

Put $A_{1}=Q_{n, 1}$. Define $\phi: A_{1} \rightarrow Q_{n}$. Since every partition in $\phi\left(A_{1}\right)$ has least part greater than 1 , we have $\phi\left(A_{1}\right) \cap A_{1}=\emptyset$. Since each partition in $A_{1}$ has identical least part, $\phi$ cannot be a many-to-one mapping. Thus, $\phi$ is an one-to-one mapping. Moreover, we see that image of every partition with even (resp. odd) number of parts in $A_{1}$ under $\phi$ has odd (resp. even) number of parts. Consequently, the number of even partitions (partitions with even number of parts) and odd partitions (partitions with odd number of parts) in $\phi\left(A_{1}\right) \cup A_{1}$ are same.

Define $A_{2}=\left(Q_{n} \backslash\left(A_{1} \cup \phi\left(A_{1}\right)\right) \cap Q_{n, 2}\right.$. Consider the mapping $\phi: A_{2} \rightarrow Q_{n}$. Following the line of argument in the last paragraph, we again get that $\phi\left(A_{2}\right) \cap A_{2}=\emptyset$ and the number of even partitions and odd partitions in $\phi\left(A_{2}\right) \cup A_{2}$ are same.

For $k \geq 3$, define $A_{k}=\left(Q_{n} \backslash \cup_{i=1}^{k-1}\left(A_{i} \cup \phi\left(A_{i}\right)\right)\right) \cap Q_{n, k}$. We see that there is no possibility for the existence of a distinct partition say $\pi_{2}$ such that $\pi_{2} \in A_{r}$ and $\phi\left(\pi_{2}\right) \in \phi\left(A_{l}\right)$ for some 
$l<r$. For otherwise, there will be a distinct partition say $\pi_{1}$ such that $\phi\left(\pi_{1}\right)=\phi\left(\pi_{2}\right)$ with $\pi_{1} \neq \pi_{2}$. This gives $\left(a_{1}+1, a_{2}+1, \ldots, a_{l}+1, a_{l+1}, \ldots, a_{k-1}\right)=\left(b_{1}+1, b_{2}+1, \ldots, b_{l}+1, b_{l+1}+\right.$ $\left.1, \ldots, b_{r}+1, b_{r+1}, \ldots, b_{k-1}\right)$, where $\pi_{1}=\left(a_{1}, \ldots, a_{k}\right)$ and $\pi_{2}=\left(b_{1}, \ldots, b_{k}\right)$ with $a_{k}=l$ and $b_{k}=r$. Consider the partition $\pi^{*}=\left(b_{1}, b_{2}, \ldots, b_{l}, b_{l+1}+1, \ldots, b_{r}+1, b_{r+1}, \ldots, b_{k-1}, b_{k}, l\right)$. From the above equality we have $b_{l+1}+1<b_{l}$ and since $l<b_{k}<k$, one can see that $\pi^{*}$ is a distinct partition of $n$ with least part $l$ such that $l$ is less than $k$. Furthermore, $\phi\left(\pi^{*}\right)=\pi_{2}$. Thus, $\pi_{2} \in \phi\left(A_{l}\right)$ which implies $\pi_{2} \notin A_{r}$, which is a contradiction.

Accordingly, we have the following conclusions:

1. $\phi\left(A_{k}\right) \cap A_{k}=\emptyset$ for every $k \in\{1,2, \ldots\}$.

2. The number of even and odd partitions in $\cup_{i \geq 1}\left(A_{i} \cup \phi\left(A_{i}\right)\right)$ are same.

Let $Q_{n}^{*}=\cup_{i \geq 1}\left(A_{i} \cup \phi\left(A_{i}\right)\right)$. A closer examination of the set $Q_{n} \backslash Q_{n}^{*}$ completes the proof. Let $\pi=\left(a_{1}, a_{2}, \ldots, a_{k}\right) \in Q_{n} \backslash Q_{n}^{*}$. Define $c(\pi)$ to be the largest integer $l \geq 2$ for which $a_{1}, a_{2}, \ldots, a_{l}$ satisfies $a_{2}-a_{1}=a_{3}-a_{2}=\cdots=a_{l}-a_{l-1}=1$. We claim that $c(\pi)=k$. For if $c(\pi)=s$ for some $s<k$, then it is plain that we can write $\pi=\left(b, b-1, \ldots, b-(s-1), a_{s+1}, \ldots, a_{k}\right)$ with $(b-(s-1))-a_{s+1}>1$. Now consider the partition $\pi_{1}=\left(b-1, b-2, \ldots, b-s, a_{s+1}, \ldots, a_{k}, s\right)$. From the membership of $\pi$, we have $a_{k} \geq k$. Since $s<k$, we have $a_{k}-s>0$. Thus $\pi_{1}$ is a distinct partition of $n$. Also, we have $\phi\left(\pi_{1}\right)=\pi$. If $\pi_{1} \in A_{i}$ for some $i$, then we have $\pi \in \phi\left(A_{i}\right)$, which leads to the conclusion that $\pi \in Q_{n}^{*}$ which is a contradiction. On the other hand, if $\pi_{1} \in \phi\left(A_{j}\right)$ for some $j$, then there exist a distinct partition say $\pi_{2}=\left(b_{1}, b_{2}, \ldots, b_{k+2}\right)$ such that $\phi\left(\pi_{2}\right)=\pi_{1}$. Note that $1 \leq b_{k+2}<s$. From this it follows that $1 \leq b_{k+2}<k$ and $b_{k+2}<s$. Since $\phi\left(\pi_{2}\right)=\pi_{1}$, we have the following equalities: $b_{1}+1=b-1, b_{2}+1=b-2, \ldots, b_{b_{k+2}}+1=b-b_{k+2}, b_{b_{k+2}+1}=$ $b-b_{k+2}-1, \ldots$; which leads to the equality $b_{b_{k+2}}-b_{b_{k+2}+1}=0$ which is a contradiction. Thus $c(\pi)=k$. Accordingly, $\pi$ is of the form $\pi=\left(a_{k}+k-1, a_{k}+k-2+, \ldots, a_{k}+1, a_{k}\right)$.

We claim that $a_{k}$ can assume only two values namely $k$ or $k+1$. From the membership of $\pi$ it follows that $a_{k} \geq k$. Suppose that $a_{k}>k+1$. Then consider the partition $\pi_{1}=$ $\left(a_{k}+(k-2), \ldots, a_{k}, a_{k}-1, k\right)$. Clearly, $\pi_{1}$ is a distinct partition of $n$. We see that $\phi\left(\pi_{1}\right)=\pi$, which implies that, $\pi_{1} \notin Q_{n} \backslash Q_{n}^{*}$. This in turn implies that $\pi_{1} \in Q_{n}^{*}$. If $\pi_{1} \in A_{i}$ for some $i$, then we would have $\phi\left(\pi_{1}\right) \in \phi\left(A_{i}\right)$, that is, $\pi \in Q_{n}^{*}$ which is a contradiction. If $\pi_{1} \in \phi\left(A_{j}\right)$ for some $j$, then there will be a distinct partition of $n$ say $\pi_{2}=\left(b_{1}, b_{2}, \ldots, b_{k+2}\right)$ such that $\phi\left(\pi_{2}\right)=\pi_{1}$. Now we make it a point that $b_{k+2}<k$. Since $\phi\left(\pi_{2}\right)=\pi_{1}$, we have the equalities $b_{1}+1=a_{k}+(k-2), b_{2}+1=a_{k}+(k-3), \ldots, b_{b_{k+2}}+1=a_{k}+(k-1)-b_{k+2}, b_{b_{k+2}+1}=$ $a_{k}+(k-1)-\left(b_{k+2}+1\right), \ldots ;$ this gives $b_{b_{k+2}}=b_{b_{k+2}+1}$, which is absurd. Thus the claim follows.

From these observations, we get that $r_{e}(n)-r_{o}(n)=0$ if $n$ is not of the forms: $k+(k+1)+$ $\cdots+(k+(k-1))$ and $(k+1)+(k+2)+\cdots+(k+k)$, that is, when $n \neq \frac{3 k^{2} \pm k}{2}$. On the other hand, if $n=\frac{3 k^{2} \pm k}{2}$ then we have $r_{e}(n)-r_{o}(n)=1$ when $k$ is even, and $r_{e}(n)-r_{o}(n)=-1$ when $k$ is odd.

This completes the proof. 


\section{References}

[1] Andrews, G. E. (1965) A Simple proof of Jacobi's Triple Product Identity, Proceedings of the American Mathematical Society, 16, 2, 333-334.

[2] Andrews, G. E. (1984) Generalised Frobenius partitions, Mem. Amer. Math Soc., 49, 301, iv+44 pp.

[3] Cheema, M. S. (1964) Vector partitions and Combinatorial identities, Math. Comp., 18, 414-420.

[4] Dyson, F. J. (1969) A new symmetry of partitions, J. Combin. Theory, 7, 56-61.

[5] Euler, L. (1780) Evolution producti infiniti $(1-x)\left(1-x^{2}\right)\left(1-x^{3}\right)\left(1-x^{4}\right)\left(1-x^{5}\right)$ etc. in seriem simplicem, Acta Academiae Sci entarum Imperialis petropolitinae 1780, 1783, $47-55$.

[6] Ewell, J. A. (1983) A Simple Proof of Fermat's Two-Square Theorem, Amer. Math. Monthly, 90, 9, 635-637.

[7] Franklin, F. (1881) Surle dévelopment du produit infini $(1-x)\left(1-x^{2}\right)\left(1-x^{3}\right)\left(1-x^{4}\right) \ldots$, Comptes Rendus Acad. Sci. Paris, 92, 448-450.

[8] Hirschhorn, M. D. (1985) A Simple Proof of Jacobi's Two-Square Theorem, Amer. Math. Monthly, 92, 8, 579-580.

[9] Sudler, C. (1966) Two enumerative proofs of an identity of Jacobi, Proc. Edinburgh. Math. Soc., 15, 67-71.

[10] Wei, C., Gong, D. (2011) Euler's pentagon number theorem implies Jacobi triple product identity, Integers, 11, 6, 811-814.

[11] Wright, E. M. (1965) An enumerative proof of an identity of Jacobi, J. London Math. Soc., $40,55-57$. 